\title{
Erratum: Constraints on dark photons and axionlike particles from the SuperCDMS Soudan experiment [Phys. Rev. D 101, 052008 (2020)]
}

\author{
T. Aralis et al.* \\ (SuperCDMS Collaboration)
}

(Received 11 January 2021; published 5 February 2021)

DOI: 10.1103/PhysRevD.103.039901

There is an error in the implementation of the limit setting method for the iZIP datasets in our publication setting constraints on dark photon and axionlike particle dark matter.

The signal window is defined as $\pm 1 \sigma$ about the dark matter mass equivalent energy, where $\sigma$ is the resolution at that energy. The Poisson upper $90 \%$ confidence limit on the number of events in the signal window was converted to an upper limit on the event rate by dividing by the efficiency-weighted exposure. The efficiency factor is calculated by integrating the product of the signal model and the energy-dependent efficiency over the signal window. For the iZIP datasets, the average efficiency over the signal window was mistakenly used, ignoring the effect that approximately $32 \%$ of the signal is lost due to the limited window size. The efficiency factors were therefore too large by a factor of approximately 1.47.

This error has no impact on the limits for dark matter masses below $3 \mathrm{keV} / c^{2}$ and therefore does not affect the region in which we claim to set the strongest laboratory constraints.

Additionally, in the original Figs. 8, 9, and 10, there is a minor inconsistency (but not inaccuracy) in the visualization, as the precise mass values for which the limits are shown vary slightly between the figures. The limits are calculated for a dense set of masses; for better clarity in the figures, the limit curves are downsampled by a factor of 50, but unintentionally, a different subset of masses was picked for each figure. The three figures have now been made consistent, all using the masses shown in the original Fig. 9.

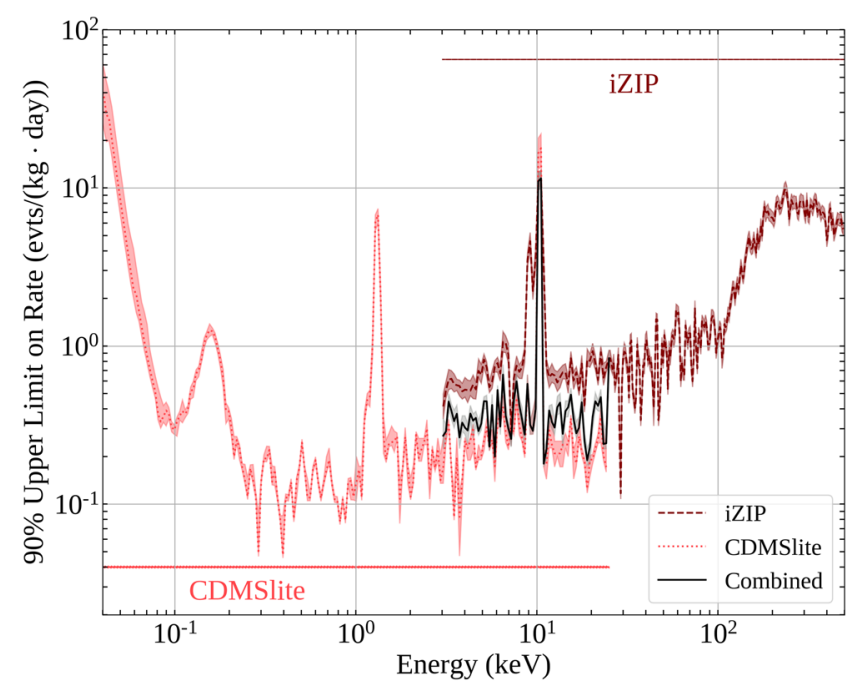

FIG. 1. Corrected calculated rates from combining data from the CDMSlite runs (light red dotted line), the iZIP detectors (dark red dashed line), and all datasets (black solid line). The shaded region around each line corresponds to the uncertainty on the rate limits. The elevation in the limits near $10 \mathrm{keV}, 1 \mathrm{keV}, 160 \mathrm{eV}$ and in iZIP data around $9 \mathrm{keV}$ results from the higher measured rates due to the Ge K-, $\mathrm{L}-$, and $\mathrm{M}$-captures and the K-capture of ${ }^{65} \mathrm{Zn}$, respectively.

"Full author list given at the end of the original paper. Published by the American Physical Society under the terms of the Creative Commons Attribution 4.0 International license. Further distribution of this work must maintain attribution to the author(s) and the published articles title, journal citation, and DOI. 


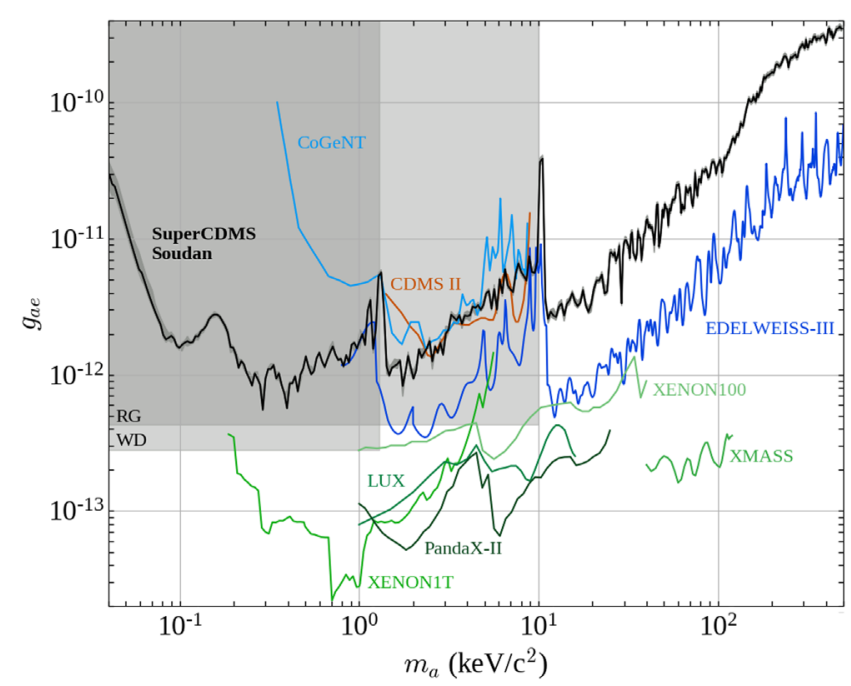

FIG. 2. Corrected SuperCDMS Soudan upper limit (solid black) with uncertainty band (shaded gray) on the axioelectric coupling. Also shown are limits set by other direct-detection experiments including: CDMS II [1], CoGeNT [2], EDELWEISS-III [3], LUX [4], PandaX-II [5], XENON100 [6], XENON1T [7], and XMASS [8]. The shaded regions are excluded by the observed cooling of red giant (RG) $[9,10]$ and white dwarf (WD) stars $[10,11]$.

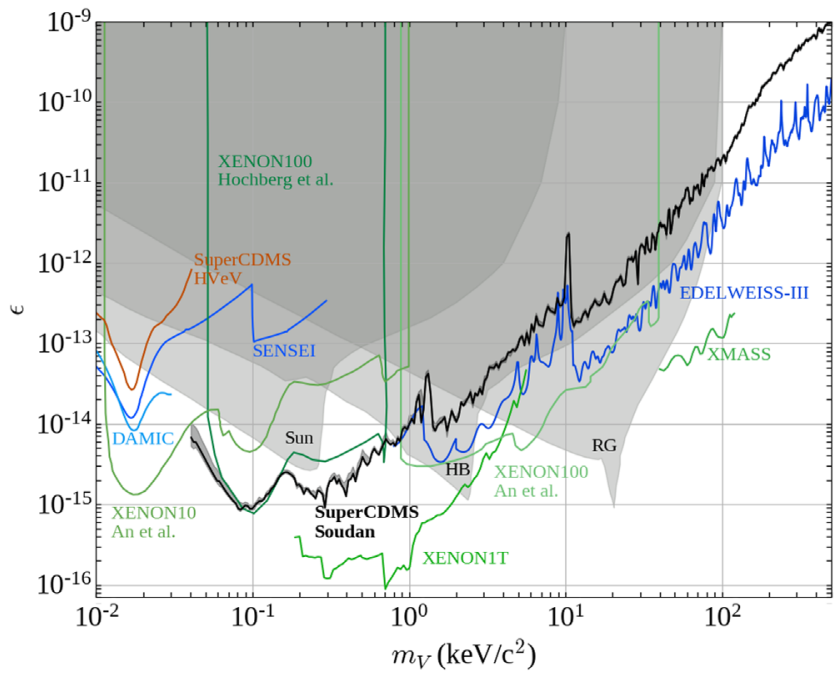

FIG. 3. Corrected SuperCDMS Soudan upper limit (solid black) with uncertainty band (shaded grey) on the dark photon's kinetic mixing. Also shown are limits set by other direct-detection experiments including DAMIC [12], EDELWEISS-III [3], SENSEI [13], the SuperCDMS HVeV device [14], An et al.'s analysis of XENON10 and XENON100 [15], and Hochberg et al.'s analysis of XENON100 [16], XENON1T [7], and XMASS [8]. The shaded regions show limits set from anomalous energy loss mechanisms in the Sun, horizontal branch stars (HB), and red giants (RG) from Ref. [15].

Updated versions of the original Figs. 8, 9, and 10 are provided in this erratum as Figs. 1, 2, and 3, respectively.

[1] Z. Ahmed et al. (SuperCDMS Collaboration), Phys. Rev. Lett. 103, 141802 (2009).

[2] C. E. Aalseth et al. (CoGeNT Collaboration), Phys. Rev. Lett. 101, 251301 (2008); 102, 109903(E) (2009).

[3] E. Armengaud et al. (EDELWEISS Collaboration), Phys. Rev. D 98, 082004 (2018).

[4] D. S. Akerib et al. (LUX Collaboration), Phys. Rev. Lett. 118, 261301 (2017).

[5] C. Fu et al. (PandaX Collaboration), Phys. Rev. Lett. 119, 181806 (2017).

[6] E. Aprile et al. (XENON100 Collaboration), Phys. Rev. D 90, 062009 (2014); 95, 029904 (2017).

[7] E. Aprile et al. (XENON Collaboration), Phys. Rev. Lett. 123, 251801 (2019).

[8] K. Abe et al. (XMASS Collaboration), Phys. Lett. B 787, 153 (2018). 
[9] N. Viaux, M. Catelan, P. B. Stetson, G. G. Raffelt, J. Redondo, A. A. R. Valcarce, and A. Weiss, Phys. Rev. Lett. 111, 231301 (2013).

[10] M. Tanabashi (Particle Data Group), Phys. Rev. D 98, 030001 (2018).

[11] I. M. Bloch, R. Essig, K. Tobioka, T. Volansky, and T.-T. Yu, J. High Energy Phys. 06 (2017) 087.

[12] A. Aguilar-Arevalo et al. (DAMIC Collaboration), Phys. Rev. Lett. 123, 181802 (2019).

[13] O. Abramoff et al. (SENSEI Collaboration), Phys. Rev. Lett. 122, 161801 (2019).

[14] R. Agnese et al. (SuperCDMS Collaboration), Phys. Rev. Lett. 121, 051301 (2018); 122, 069901(E) (2019).

[15] H. An, M. Pospelov, J. Pradler, and A. Ritz, Phys. Lett. B 747, 331 (2015).

[16] Y. Hochberg, T. Lin, and K. M. Zurek, Phys. Rev. D 95, 023013 (2017). 